\title{
Determination of Radio Environmental Level in Hydrogen (HI) Band Spectrum by Using Kurtosis Analysis
}

\author{
Sharifah Nurul Aisyah Syed Zafar\#, Roslan Umar ${ }^{\#}$, Nor Hazmin Sabri ${ }^{*}$, Zainol Abidin Ibrahim ${ }^{\#}$ Mohd Khairul Amri \\ Kamarudin", Azizah Endut ${ }^{\#}$ \\ ${ }^{\#}$ East Coast Environmental Research Institute, Universiti Sultan Zainal Abidin, Gong Badak, 21300 Kuala Nerus, Terengganu, Malaysia \\ E-mail:sharifahaisyah95@yahoo.com; roslan@unisza.edu.my; mkhairulamri@unisza.edu.my; enazizah@unisza.edu.my \\ *School of Fundamental Science, Universiti Malaysia Terengganu, 21030 Terengganu, Malaysia \\ E-mail: norhazmin@umt.edu.my \\ ${ }^{\#}$ Department of Physics, University of Malaya, 50603 Kuala Lumpur, Malaysia \\ E-mail:drzai@um.edu.my
}

\begin{abstract}
The Radio Frequency Interference (RFI) nowadays becomes a serious problem and crucial issue in the radio astronomy observation. The radio astronomical lines observation become worse due to the more RFI in Ultra High Frequency (UHF) and Very High Frequency (VHF) which influences the data in radio image. The aims of this paper are to investigate the radio environment level in hydrogen (HI) band $(1300-1500 \mathrm{MHz})$ and also in a wide band $(0-9000 \mathrm{MHz})$. The observation site is at KUSZA Observatory (KO), Langkawi National Observatory (LNO) and East Coast Environmental Research Institute (ESERI). In this paper, the statistical kurtosis and waterfall method are applied on the radio environment data. The highest average of kurtosis values indicates the lowest of radio environment level at these sites. From obtained results, the highest average of kurtosis value in wide band obtained at ' $L N O$ ' which 20.33 and in the specific band was found at 'KO' with a value of 6.06. This preliminary study is important to identify the radio environment level in radio astronomical specific band such as $\mathrm{HI}$ band.
\end{abstract}

Keywords - radio astronomy; radio frequency interference; kurtosis analysis; hydrogen band spectrum

\section{INTRODUCTION}

Radio Astronomy is a growing, young and vigorous science in which it is being explored in many parts of the universe and has been making a lot of new discoveries. To name some examples of a celestial object captured; quasars, pulsars, the Big Bang and many phenomena were first revealed by radio astronomer [1], [2]. In radio astronomy observation, the celestial object is studied by capturing the radio waves [19] at all frequency emitted from the celestial within electromagnetic radiation.

The characteristic of frequency depends on the intensity of the emission which based on the physical condition and characteristic of radio source [3]. A radio telescope is used as the instrument to listen or receive radio waves from the outer spaces. The larger area of antenna used the higher the sensitivity of radio telescope in detecting the radio frequencies [4], [5]. However, major issues constantly exist in observing radio astronomical lines and the problem is radio frequency interferences.
Therefore, the RFI studies were done in order to determine the sites of low RFI level for radio telescope location. Generally, the interferences usually come from man-made interferences and also natural weather. Examples of man-made interferences include a communication system, radar, electronic equipment, $\mathrm{AM} / \mathrm{FM}$ radio and service provider [6]. Several users with their frequency allocation by Malaysian Communication Multimedia Commission (MCMC) and International Telecommunication Union (ITU) was presented in Table 1.

The weather factors such as rain, the wind, and solar radiation can also give impacts to the radio frequency. In fact, many previous studies on RFI investigation for astronomical lines observation have been done. From the studies, sources of RFI, sites of low RFI and free RFI spectrum band for radio astronomy observation could be determined.

According to [7], [8], they found that the major effect of RFI on astronomical lines is below $1 \mathrm{GHz}$. The study was done in Malaysia and Thailand, and it was found that the 
interferences come from electronic equipment especially radio navigation $(731 \mathrm{MHz}$ until $752 \mathrm{MHz})$, radio broadcasting (151 MHz, $151.8 \mathrm{MHz}$ and $152 \mathrm{MHz}$ ), aeronautical navigation $(245.5 \mathrm{MHz}, 248.7 \mathrm{MHz}$ and 249 $\mathrm{MHz})$ and fixed mobile $(605 \mathrm{MHz}, 608.3 \mathrm{MHz}, 612.2 \mathrm{MHz}$ and $613.3 \mathrm{MHz}$ ). At the same time, it was observed that the frequency band of $320 \mathrm{MHz}$ until $330 \mathrm{MHz}$ were free from interferences and best band to conduct the solar burst observation [7], [8], [20]. Several radio astronomical lines with their own rest frequency in the radio band as listed by MCMC and ITU were shown in this paper. The details are tabulated in Table 1.

TABLE I

SEVERAL User AND THEIR FReQUENCy ALLOCATION As Listed By MCMC AND ITU [9]

\begin{tabular}{|l|l|l|}
\hline Frequency (MHz) & ITU Allocation & MCMC Allocation \\
\hline $312-315$ & Fixed mobile, mobile satellite (Earth to space) & Fixed mobile, mobile satellite (Earth to space) \\
\hline $315-322$ & Fixed mobile & Fixed mobile \\
\hline $322-328.6$ & Fixed mobile, radio astronomy & Fixed mobile, radio astronomy \\
\hline $328.6-335.4$ & Aeronautical, radio navigation & Aeronautical, radio navigation \\
\hline $400.05-400.15$ & Standard frequency and time signal-satellite & Standard frequency and time signal-satellite, fixed mobile \\
\hline $402-403$ & $\begin{array}{l}\text { Meteorological aids, earth exploration-satellite(earth- } \\
\text { to-space), meteorological-satellite (earth-to-space), } \\
\text { fixed mobile except aeronautical mobile }\end{array}$ & $\begin{array}{l}\text { Meteorological aids, earth exploration-satellite (earth-to- } \\
\text { space), meteorological-satellite (earth-to-space), fixed } \\
\text { mobile except aeronautical mobile }\end{array}$ \\
\hline $406.1-410$ & $\begin{array}{l}\text { Fixed mobile except (aeronautical mobile), } \\
\text { radio astronomy }\end{array}$ & $\begin{array}{l}\text { Fixed mobile except (aeronautical mobile), } \\
\text { radio astronomy }\end{array}$ \\
\hline $438-440$ & Amateur, radiolocation & $\begin{array}{l}\text { Fixed mobile except (aeronautical mobile), amateur, } \\
\text { radiolocation }\end{array}$ \\
\hline $585-610$ & Fixed mobile, broadcasting, radio navigation & Fixed mobile, broadcasting, radio navigation \\
\hline $614-698$ & Broadcasting, fixed mobile & Broadcasting, mobile \\
\hline $790-806$ & $\begin{array}{l}\text { Fixed Broadcasting, mobile except (aeronautical } \\
\text { mobile), fixed mobile }\end{array}$ & Fixed mobile, broadcasting \\
\hline $862-890$ & Fixed mobile, broadcasting & Fixed mobile, broadcasting \\
\hline $928-942$ & $\begin{array}{l}\text { Fixed mobile except (aeronautical mobile), } \\
\text { radiolocation }\end{array}$ & Fixed mobile, radiolocation \\
\hline $960-1164$ & Aeronautical radio navigation, aeronautical mobile & Aeronautical radio navigation, aeronautical mobile \\
\hline
\end{tabular}

TABLE III

The Rest FreQuenCy AND SugGested BANDWIDTH OF ASTRONOMICAL LiNES [10], [11]

\begin{tabular}{|l|l|l|l|}
\hline Atom/Molecule & Lines Name & Rest Frequency (MHz) & Suggested Minimum Bandwidth (MHz) \\
\hline DI & Deuterium & 327.384 & 327.0 to 327.7 \\
\hline HI & Neutral hydrogen & 1420.406 & 1370 to 1427 \\
\hline OH & Hydroxyl radical & 1612.231 & 1610.6 to 1613.8 \\
\hline OH & Hydroxyl radical & 1665.402 & 1660 to 1670 \\
\hline OH & Hydroxyl radical & 1667.359 & 1718.8 to 1722.2 \\
\hline OH & Hydroxyl radical & 1720.530 & 3260.0 to 3267.0 \\
\hline
\end{tabular}

The focus of this study is to identify the radio environment level in the specific band between $1300 \mathrm{MHz}$ until $1500 \mathrm{MHz}$ which presented as $\mathrm{HI}$ band at the three selected sites. This band is important because the frequency of hydrogen spectral lines was at a rest frequency of $1420.406 \mathrm{MHz}$.

Besides, investigation of the radio environment level in a wide band $(0-9000 \mathrm{MHz})$ also has been done. As for the analysis method, the basic statistical kurtosis method was also applied by calculating the average of kurtosis value at every selected site. A positive sign of kurtosis value indicates leptokurtic which mean the peak is too tall and small standard deviation, whereas the negative sign of kurtosis shows platykurtic. During this condition, the peak is too flat and high in standard deviation [12], [13].

Thus, the breadth or narrowness of interference peak in HI band can be identified by computing the kurtosis value. Fig. 1 and 2 illustrates the notion of kurtosis. In our case, the wider bandwidth of nearby interference peak in HI band, the fewer potential nearby interferences are able to enter $\mathrm{HI}$ specific band which is set at $1370-1427 \mathrm{MHz}$ by ITU.
As a result, lower average kurtosis value was computed. Besides, the average for radio environment level is also calculated in HI band at selected sites in order to strengthen the analysis. In addition to this method, we also applied the waterfall-style method to monitor the consistency of RFI in HI band well.

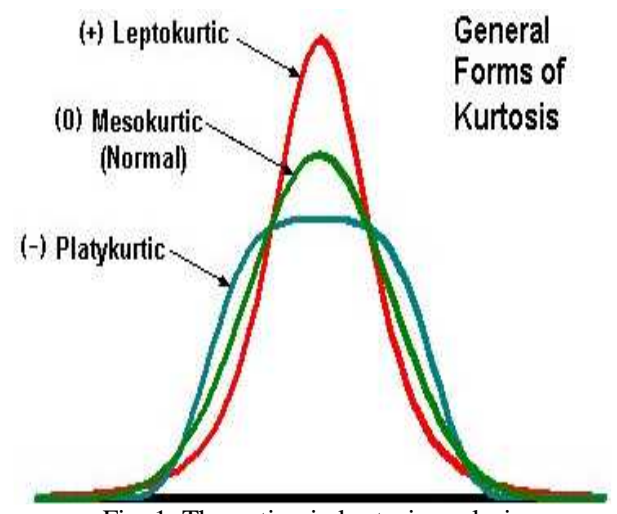

Fig. 1 The notion in kurtosis analysis 


\section{MATERIAL AND METHOD}

A receiver system is comprised of $9000 \mathrm{MHz}$ spectrum analyzer (Keysight N9915A, USA) and a copper antenna connected to Low Noise Amplifier (LNA) using Belden cable. This is so to enable the measurement of the radio environment level in a wideband (up to $9000 \mathrm{MHz}$ ) and $\mathrm{HI}$ band $(1300-1500 \mathrm{MHz})$. Fig. 3 shows the receiver system setup. The observation was done in 4 hours in the oneminute interval at each three selected sites; KUSZA Observatory (KO), Langkawi National Observatory (LNO) and East Coast Environmental Research Institute (ESERI).

These sites are chosen due to sites location in a lowdensity population area of which the use of low RFI is possible. These locations are considered as ideal sites for locating radio telescope and conducting radio astronomical observation as the criterion of locating the radio telescope a far away as possible from man-made interferences, is met.

The description of selected sites is shown in Table 3. The measured radio environment spectrum level then was analyzed by plotting a graph of power level against frequency for the wide and HI band in order to observe the radio environment level between three selected sites. The data is further analyzed by presenting the kurtosis value from the data and supported by averaging the radio environment spectrum at each site in HI band in order to compare with sites which have low RFI

This kurtosis value such as 'indicator' of radio environment level was used in this analysis at many sites as referred to [14]. The highest in kurtosis value obtained indicates the lowest radio environment level. As such, the average of kurtosis value was computed in order to indicate the radio environment level without looking at the radio spectrum graph.

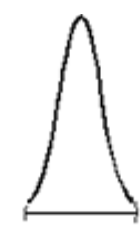

Leptokurtic distribution

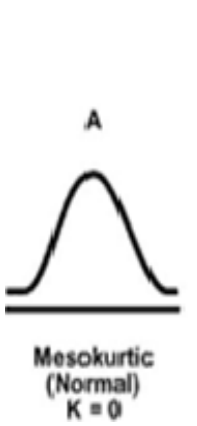

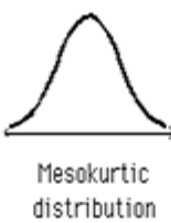

(a)

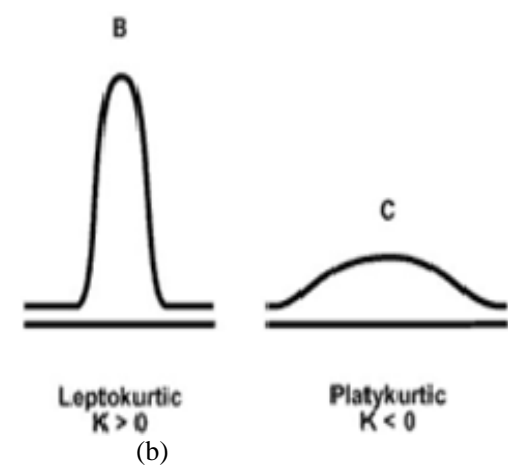

Fig. 2 Kurtosis distribution

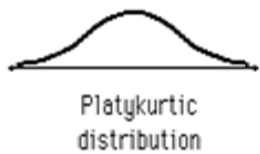

distribution
In addition, the waterfall-style method was utilized to identify the consistency of peak in radio environment spectrum in HI band at the three sites. Finally, the best site with minimum radio environment level in $\mathrm{HI}$ band was determined by computing the kurtosis value and averaging value of radio environment spectrum.

TABLE IIIII

DESCRIPTION OF SELECTED SITES

\begin{tabular}{|l|l|l|}
\hline Sites & Characteristics & Distance from Urban Area (km)/(Location) \\
\hline KO & Low density population, on top of hill & $23.9 /$ Peninsular Malaysia (East Coast) \\
\hline LNO & $\begin{array}{l}\text { Low density population, located in remote area, nearby Royal Navy } \\
\text { Malaysian (RMN) }\end{array}$ & $10.5 /$ Langkawi Island) \\
\hline ESERI & High density population, located in campus of university & $15 /$ Peninsular Malaysia (East Coast) \\
\hline
\end{tabular}

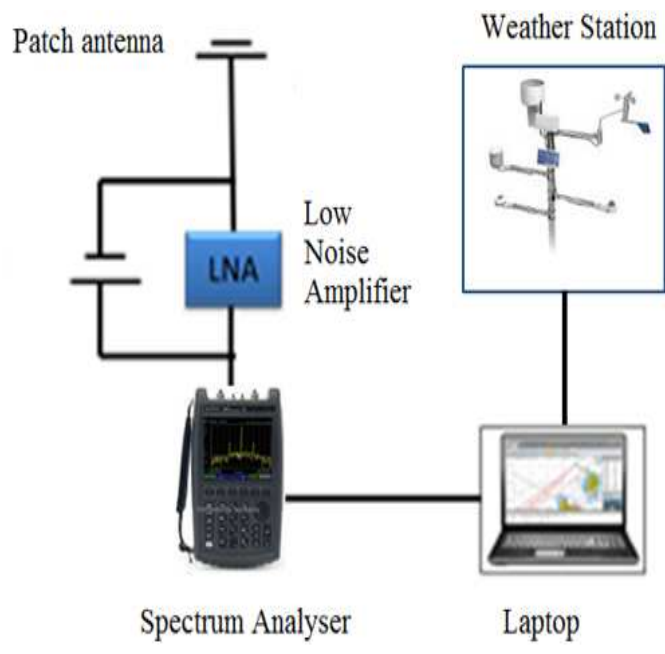

Fig. 3 Receiver system setup [15], [16], [17]

\section{RESULTS AND DISCUSSION}

The radio environment spectrum for the three selected sites in the wide band is shown in Fig. 4 and 5. Based on the graph, the lowest radio environment level is observed at LNO as the lower in fluctuations is seen as compared to another two sites.

To add to this analysis, the kurtosis value was calculated to determine the radio environment level at these three sites. Using statistical kurtosis method, 'LNO' shows the highest kurtosis value of 20.23, while in 'KO' the kurtosis value was recorded at 18.33 and 14.69 for 'ESERI'. The equation of kurtosis is presented as below. For references, the radio-free zone should possess the kurtosis value above 10 .

'LNO' was found as the best site of low radio environment level with kurtosis value above 10 in the wide band [14]. At the same time, it was also observed that low-density population and distant location from urban area contributed to the low in RFI at 'LNO' in the wide band. 
Kurtosis:

$$
\mathrm{a}_{4}=\mathrm{m}_{4} / \mathrm{m}_{2}^{2}
$$

where

$$
\mathrm{m}_{4}=\sum(\mathrm{x}-\overline{\mathrm{x}})^{4} / \mathrm{n} \text { and } \mathrm{m}_{2}=\sum(\mathrm{x}-\overline{\mathrm{x}})^{2} / \mathrm{n}
$$

In this paper, research focused on the investigation of radio environment level in HI band (1300-1500 MHz). From the results, it can be concluded that the best site of low radio environment level is at 'KO' in $\mathrm{HI}$ band with 6.06 average kurtosis value. Since the kurtosis value is positive or leptokurtic, the interference peak forms the tall and narrow width shape. It was clearly shown in Fig. 4 that the tall and narrow peak was found at ' $\mathrm{KO}$ ' in $\mathrm{HI}$ band nearby the $\mathrm{HI}$ specific band (1370-1427MHz). This tall and narrow peak owned by the frequency of $1440 \mathrm{MHz}$ and the frequency is indicated as fixed mobile by MCMC. This interference peak which was nearby HI specific band shows the lowest in width if compared to another nearby frequency interferences.

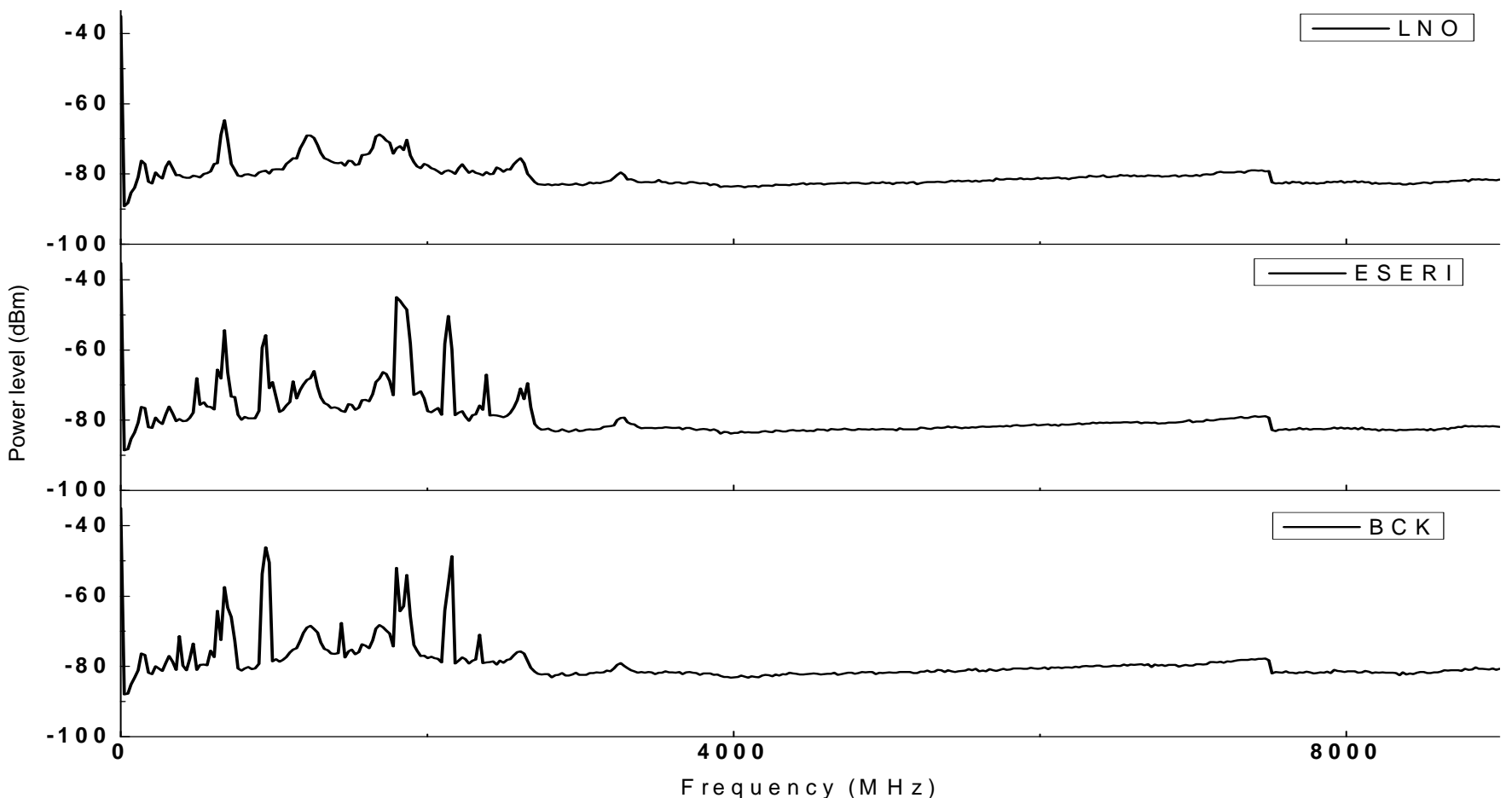

Fig. 4 The radio environment spectrum in wide band at three selected sites i) KO ii) ESERI iii) LNO

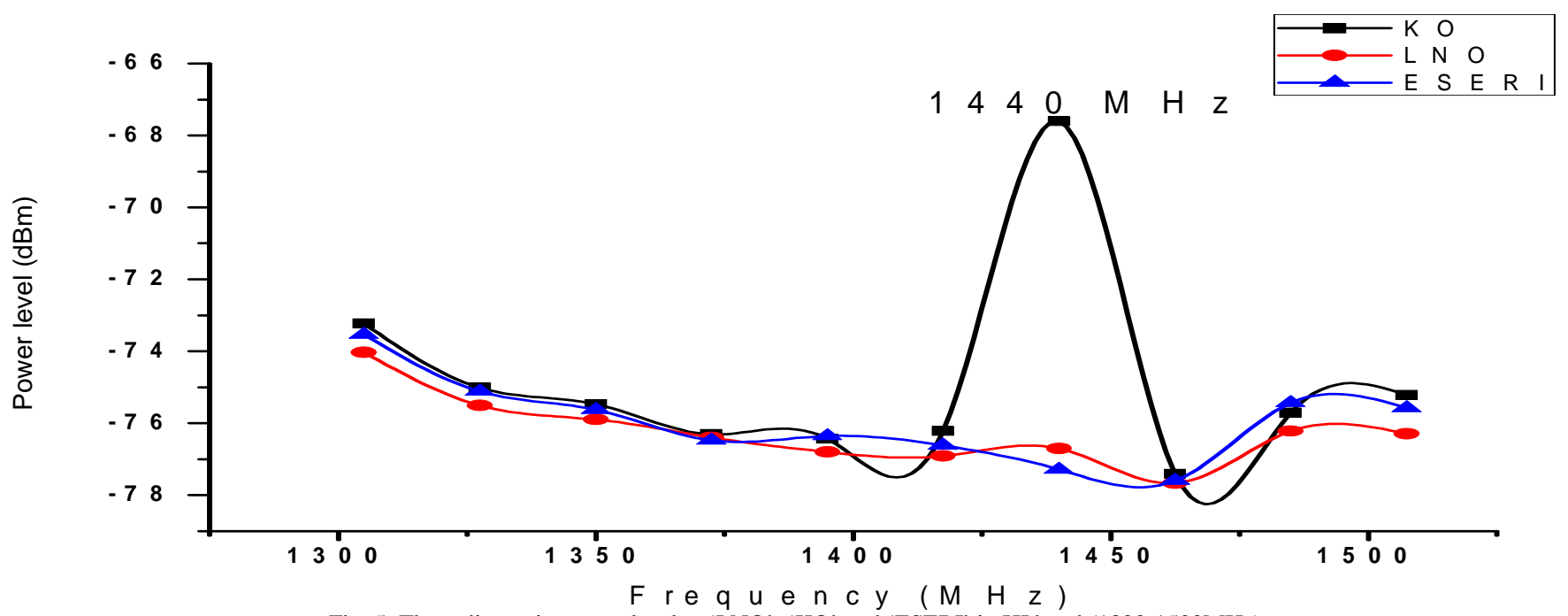

Fig. 5 The radio environment level at 'LNO', 'KO' and 'ESERI' in HI band (1300-1500MHz)

For that, it causes small interferences to enter the HI specific band. Therefore, the low radio environment level in $\mathrm{HI}$ band was indicated at 'KO'. This is different from the high width of interference peak that able to enter the HI specific band region easily due to a large area of the curve until it had contaminated band. Not only that, the low in standard deviation value in $\mathrm{HI}$ band at ' $\mathrm{KO}$ ' as compared to another two sites also gave a strong evidence to the wide and narrow bell-shaped interference curve. 
The interpretation of standard deviation indicates the larger the value of standard deviation, the larger the curve to flat due to more data spread out. In the following part, the average values of radio environment spectrum at three sites are also observed in order to strengthen the analysis.

Based on the observation, the lowest average value of radio environment spectrum between the sites was found at 'KO'. It seems that the interference level was at its lowest in 'KO' if compared to other two sites. Since 'KO' has the largest kurtosis value with the lowest in the average value of radio environment spectrum, we can certainly conclude that this result indicates 'KO' as the best site among the three sites for HI lines observation. In previous studies, they only focus and determine the radio environment level in Hydroxyl lines $(\mathrm{OH})$ band.

There was no recent research that conducted an investigation in HI band. Thus in this paper, we contributed the results of radio environment level at three sites in HI band. Table 4 shows the details of kurtosis value, an average of radio environment spectrum and standard deviation in $\mathrm{HI}$ band at three sites.

TABLE IVV

Average of Kurtosis Value, Radio Environment LeVel For Three Selected Sites in HI BAND

\begin{tabular}{|l|l|l|l|}
\hline Site & Kurtosis Value & Average Value of Radio Environment Spectrum $(\mathbf{d B m})$ & Standard Deviation $(\boldsymbol{\sigma})$ \\
\hline KO & 6.06 & -74.864 & 0.89 \\
\hline LNO & 2.37 & -75.963 & 1.29 \\
\hline ESERI & 1.05 & -76.245 & 1.46 \\
\hline
\end{tabular}

Fig. 6 until 8 illustrate the waterfall graph after implementing the waterfall analysis method on the radio environment spectrum data. From the graph, the consistency of RFI was observed at KO with the frequency of $1440 \mathrm{MHz}$.
This frequency was owned by fixed mobile as listed from MCMC and ITU.

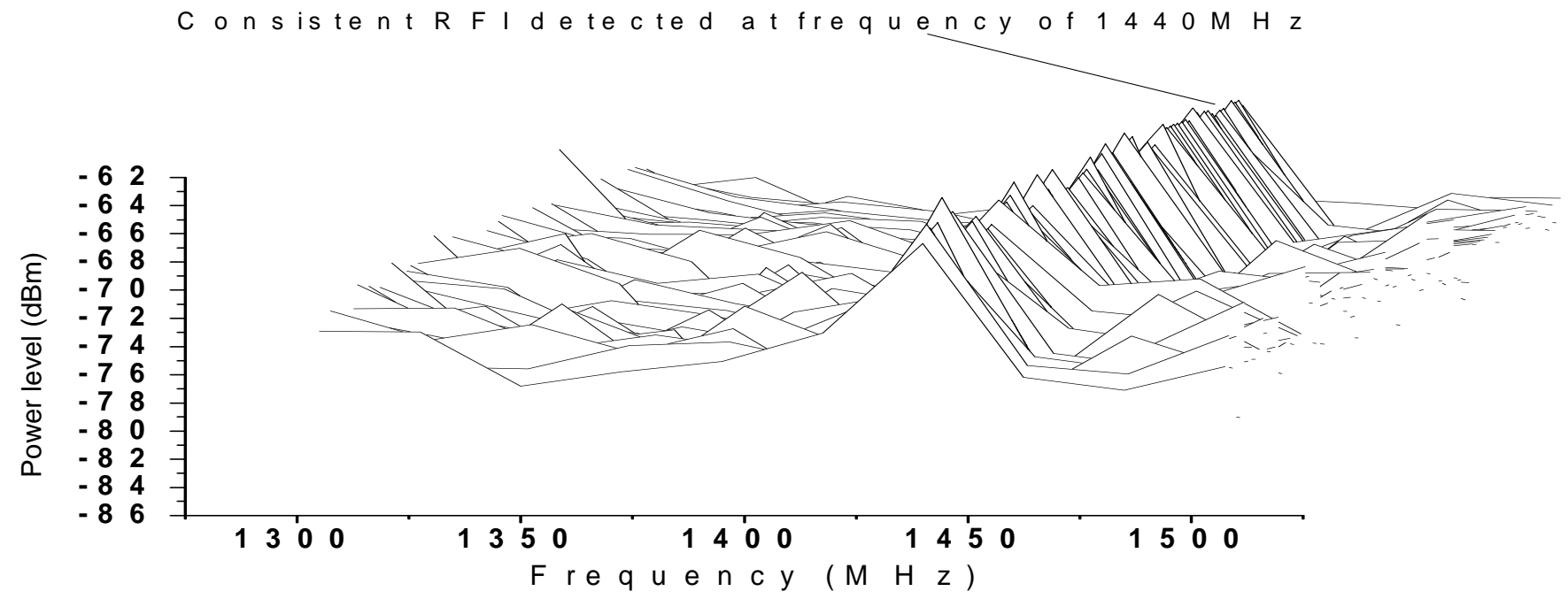

Fig. 6 Waterfall-style graph at 'KO' in $\mathrm{HI}$ band

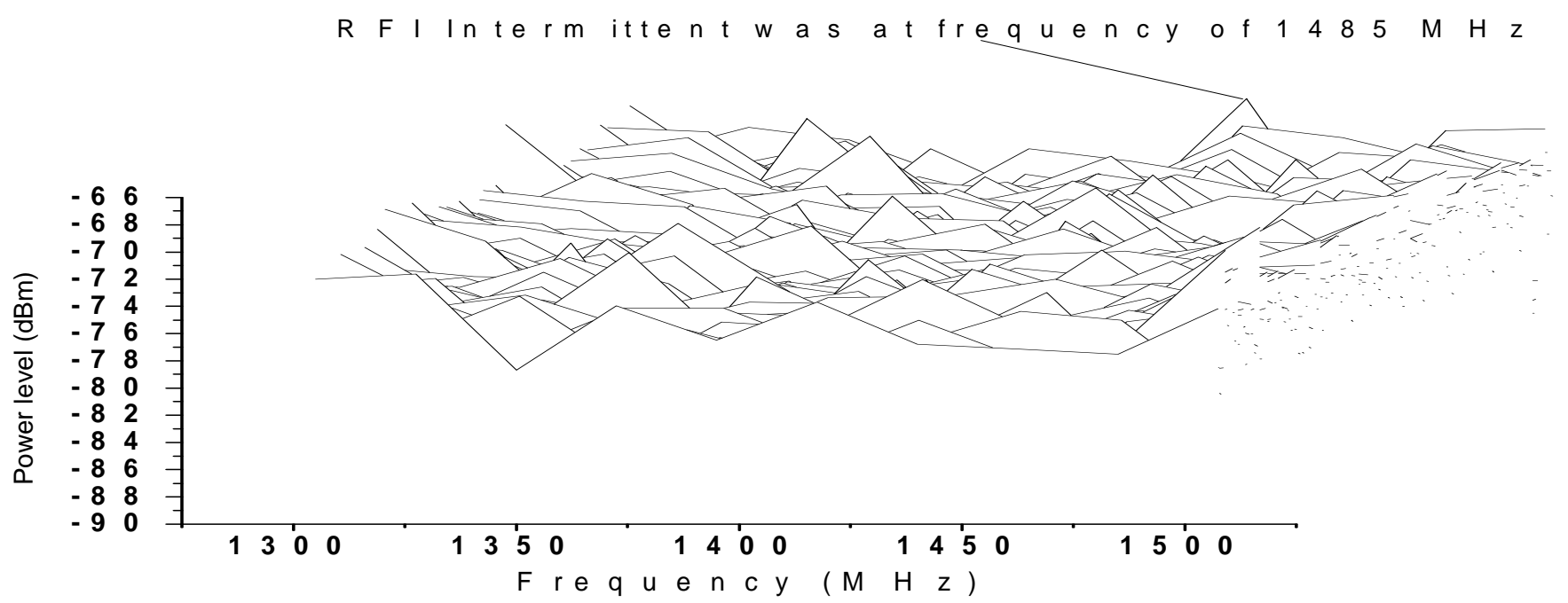

Fig. 7 Waterfall-style graph at 'LNO' in HI band 


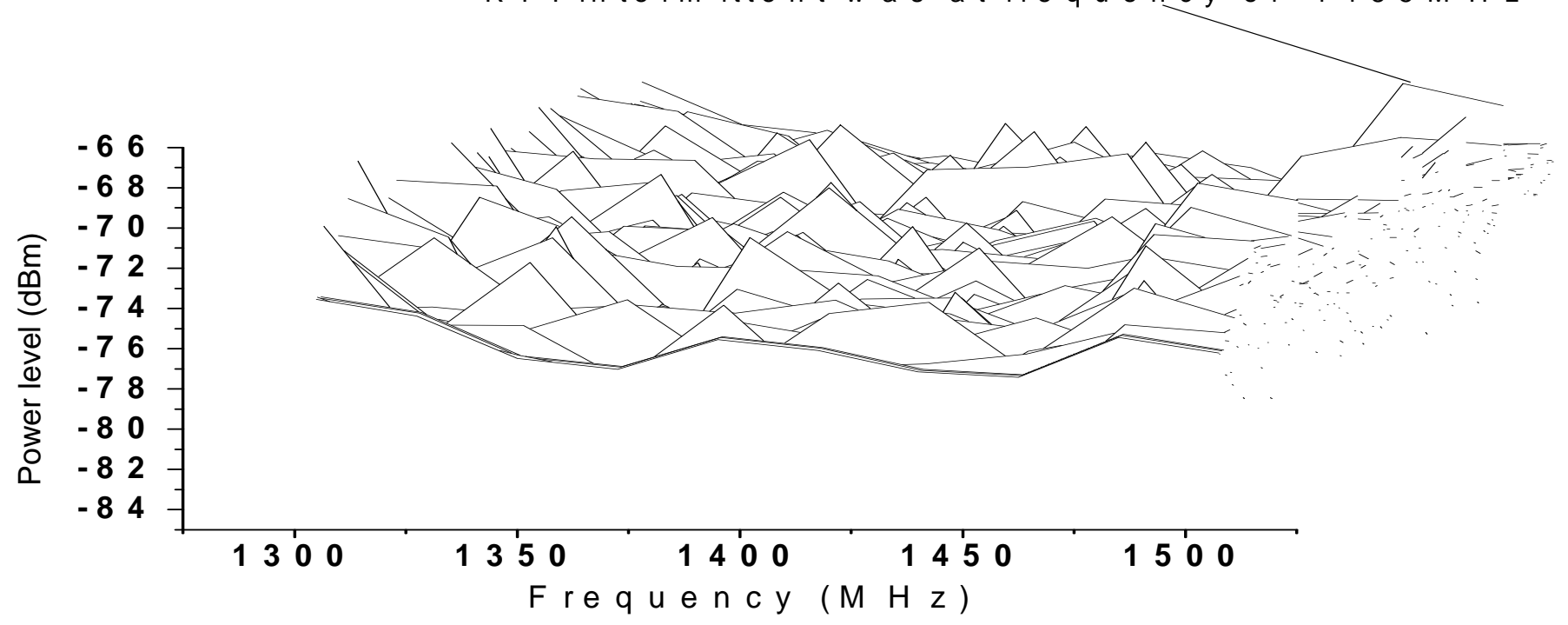

Fig. 8 Waterfall-style graph at 'ESERI' in HI band

Whereas, the RFI intermittent was observed at two sites with $1440 \mathrm{MHz}$ and $1485 \mathrm{MHz}$ frequencies recorded at LNO and ESERI respectively. This technique enables the monitoring of the consistency of RFI wisely. Besides, we are able to take action to protect the radio astronomical window.

From this research, we can conclude the 'KO' was the best site for HI spectra lines observation in Malaysia. The highest average of kurtosis value and low in average of radio environment level made the ' $\mathrm{KO}$ ' become the convenient sites for radio astronomical research due to low in radio environment level.

For future work, radio environment observation in the other astronomical lines window should be conducted in 24 hours observation. At the same time, the radio environment level should be monitored continuously in order to make sure the sites suitable for radio astronomical observation. Besides, the importance to notify the radio astronomy window to the public thereby they realize and concern the consequence. In addition, the Spectral Kurtosis (SK) estimator algorithm is needed for total RFI elimination. This algorithm is important to flag for bad data from radio image of radio telescope [18].

\section{CONCLUSION}

In this study, the 'KO' was found as the lowest radio environment level if compared to 'LNO' and 'ESERI'. The kurtosis values at three sites are 6.06, 2.37 and 1.05 representing 'KO', 'LNO' and 'ESERI' respectively. This highest of kurtosis value at $\mathrm{KO}$, in addition to the lowest radio environment spectrum level in $\mathrm{HI}$ band, was the evidence of the minimum radio environment level at KO.

\section{ACKNOWLEDGMENT}

This study is funded by the usage of the grants of Research Acculturation Collaborative Effort (RACE), project no. RACE/F1/ST1/UNISZA/15(RR118), RACE-UM (CR008/2015), FRGS/1/2015/SG02/UNISZA/02/1(RR155) and UMT-68006/INSENTIF/60. The authors would like to thanks, Universiti Sultan Zainal Abidin and Universiti Malaysia Terengganu for the financial and experimental support of this work. Special acknowledgment is dedicated to the Electromagnetic Research Group (EMRG) team members for their assistance and cooperation in assisting this work.

\section{REFERENCES}

[1] T. Sasao and A. B. Fletcher. (2005) Introduction to VLBI systems. [Online]. Available: http://www.astro.sci.yamaguchiu.ac.jp/jvn/reduction/kvnlecnote/kchap1.pdf.

[2] R. Umar, N. H. Sabri, Z. Z. Abidin, Z. A. Ibrahim, A. Azid, H. Juahir, M. E. Toriman, and M. K. A. Kamarudin, "Rain preliminary study of radio astronomical lines effect of rain below $2.9 \mathrm{GHz}$," Jurnal Teknologi, vol. 75, pp. 7-11, Jun. 2015.

[3] B. F. Burke and F. Graham-Smith, An Introduction to Radio Astronomy. 3rd ed., Cambridge, England: Cambridge University Press, 2010.

[4] N. H. Sabri, R. Umar, W. Z. A. Wan Mokhtar, Z. Zainal Abidin, Z. A. Ibrahim, A. Azid, H. Juahir, M. E. Toriman, and M. K. A. Kamarudin, "Preliminary study of vehicular traffic effect on radio signal for radio," Jurnal Teknologi, vol. 75, pp. 313-318, Jun. 2015.

[5] R. Umar, Z. Z. Abidin, A. Z. Ibrahim, N. Gasiprong, K. Asanok, S. Nammahachak, and Z. S. Hamidi, "The study of radio frequency interference (RFI) in Altitude effect on radio astronomy in Malaysia and Thailand," Middle East Journal of Scientific Research, vol. 14, pp. 861-866, 2013.

[6] J. P. G. Porko, "Radio frequency interference in radio astronomy," Master thesis, Aalto University, Helsinki, Finland, May 2011.

[7] R. Umar, S. N. Hazmin, A. Z. Ibrahim, Z. Z. Abidin, and A. Muhamad, "Measurement technique in radio frequency interference (RFI) study for radio astronomy purposes," Malaysian Journal of Analytical Sciences, vol. 19, pp. 960-965, 2015.

[8] Z. Z. Abidin, R. Umar, Z. A. Ibrahim, Z. Rosli, K. Asanok, and N. Gasiprong, "Investigation on the frequency allocation for radio astronomy at the L band," Publications of the Astronomical Society of Australia, vol. 30, pp. 47-57, Jan. 2013.

[9] Malaysian Communications and Multimedia Commission. (2006) Spectrum plan. [Online]. Available: http://www.skmm.gov.my/skmmgovmy/files/attachments/Spectrum $\%$ 20Plan_Final.pdf.

[10] J. D. Kraus, Radio Astronomy, 2nd ed., New York, USA: McGrawHill, 1986. 
[11] V. Pankonin and R. M. Price, "Radio astronomy and spectrum management: The impact of WARC-79," IEEE Transactions on Communications, vol. 29, pp. 1228-1237, Aug. 1981.

[12] H. C. Thode, Testing for Normality, 1st ed., Florida, USA: CRC Press, 2002

[13] L. T. DeCarlo, "On the meaning and use of kurtosis," Psychological Methods, vol. 2, pp. 292-307, Sep. 1997.

[14] Z. Z. Abidin, Z. A. Ibrahim, Z. Rosli, S. R. Hassan, S. F. F. Malim, N. Noorazlan, and A. S. M. Suhaimi, "Methods and applications of radio frequency interference surveys for radio astronomy in Malaysia," in Proc. IEEE IconSpace'11, 2011, p. 178.

[15] R. Umar, Z. Z. Abidin, Z. A. Ibrahim, Z. Rosli, and N. Noorazlan, "Selection of radio astronomical observation sites and its dependence on human generated RFI," Research in Astronomy and Astrophysics, vol. 14, pp. 241-248, Feb. 2014

[16] S. N. Hazmin, S. N. A. Syed Zafar, R. Umar, and W. Z. A. W. Mokhtar, "Radio frequency interference: The effect of ambient carbon dioxide $\left(\mathrm{CO}_{2}\right)$ concentration on radio signal for radio astronomy purposes," Malaysian Journal of Analytical Sciences, vol. 19, pp. 1065-1071, 2015.

[17] S. N. Hazmin, A. A. Wahidah, R. Umar, S.S. Syafa, Z. A. Ibrahim, and W. Z. A. W. Mokhtar, "Solar radiation effect on radio signal for radio astronomy purposes," Malaysian Journal of Analytical Sciences, vol. 19, pp. 1374-1381, 2015.

[18] D. E. Gary, Z. Liu, and G. M. Nita, "Erratum: A wideband spectrometer with RFI detection," Publications of the Astronomical Society of the Pacific, vol. 122, pp. 743-744, May 2010.

[19] R. Mat, M. M. Shafie, S. Ahmad, R. Umar, Y. B. Seok, and N. H. Sabri, "Temperature effect on the tropospheric radio signal strength for UHF band at Terengganu, Malaysia," International Journal on Advanced Science, Engineering and Information Technology, vol. 6, pp. 770-774, Oct. 2016.

[20] N. Z. M. Afandi, Z. Z. Abidin, R. Umar, N. H. Sabri, Z. A. Ibrahim, and C. Monstein, "Implementation of frequency drift for identification of solar radio burst type II," International Journal on Advanced Science, Engineering and Information Technology, vol. 6, pp. 775-780, Oct. 2016. 